\title{
Structure of the SARS-CoV-2 spike receptor-binding domain bound to the ACE2 receptor
}

https://doi.org/10.1038/s41586-020-2180-5

Received: 19 February 2020

Accepted: 19 March 2020

Published online: 30 March 2020

Check for updates

\author{
Jun Lan ${ }^{1,4}$, Jiwan $\mathrm{Ge}^{1,4}$, Jinfang Yu, ${ }^{1,4}$, Sisi Shan ${ }^{2,4}$, Huan Zhou ${ }^{3}$, Shilong Fan', Qi Zhang ${ }^{2}$, \\ Xuanling Shi ${ }^{2}$, Qisheng Wang ${ }^{3}$, Linqi Zhang ${ }^{2 \bowtie}$ \& Xinquan Wang ${ }^{1 凶}$
}

A new and highly pathogenic coronavirus (severe acute respiratory syndrome coronavirus-2, SARS-CoV-2) caused an outbreak in Wuhan city, Hubei province, China, starting from December 2019 that quickly spread nationwide and to other countries around the world ${ }^{1-3}$. Here, to better understand the initial step of infection at an atomic level, we determined the crystal structure of the receptor-binding domain (RBD) of the spike protein of SARS-CoV-2 bound to the cell receptor ACE2. The overall ACE2-binding mode of the SARS-CoV-2 RBD is nearly identical to that of the SARS-CoV RBD, which also uses ACE2 as the cell receptor ${ }^{4}$. Structural analysis identified residues in the SARS-CoV-2 RBD that are essential for ACE2 binding, the majority of which either are highly conserved or share similar side chain properties with those in the SARS-CoV RBD. Such similarity in structure and sequence strongly indicate convergent evolution between the SARS-CoV-2 and SARS-CoV RBDs for improved binding to ACE2, although SARS-CoV-2 does not cluster within SARS and SARS-related coronaviruses $^{1-3,5}$. The epitopes of two SARS-CoV antibodies that target the RBD are also analysed for binding to the SARS-CoV-2 RBD, providing insights into the future identification of cross-reactive antibodies.
The emergence of the highly pathogenic coronavirus SARS-CoV-2 in Wuhan and its rapid international spread has posed a serious global public-health emergency ${ }^{1-3}$. Similar to individuals who were infected by pathogenic SARS-CoV in 2003 and Middle East respiratory syndrome coronavirus (MERS-CoV) in 2012, patients infected by SARS-CoV-2 showed a range of symptoms including dry cough, fever, headache, dyspnoea and pneumonia with an estimated mortality rate ranging from 3 to $5 \%^{6-8}$. Since the initial outbreak in December of 2019, SARS-CoV-2 has spread throughout China and to more than 80 other countries and areas worldwide. As of 5 March 2020, 80,565 cases in China have been confirmed with the infection and 3,015 infected patients have died (https://www.who.int/emergencies/ diseases/novel-coronavirus-2019/situation-reports/). As a result, the epicentre Wuhan and the neighbouring cities have been under lockdown to minimize the continued spread and the WHO (World Health Organization) has announced a Public Health Emergency of International Concern owing to the rapid and global dissemination of SARS-CoV-2.

Phylogenetic analyses of the coronavirus genomes have revealed that SARS-CoV-2 is a member of the Betacoronavirus genus, which includes SARS-CoV, MERS-CoV, bat SARS-related coronaviruses (SARSr-CoV), as well as others identified in humans and diverse animal species ${ }^{1-3,5}$. Bat coronavirus RaTG13 appears to be the closest relative of the SARS-CoV-2, sharing more than $93.1 \%$ sequence identity in the spike $(S)$ gene. SARS-CoV and other SARSr-CoVs, however, are distinct from SARS-CoV-2 and share less than $80 \%$ sequence identity ${ }^{1}$.

Coronaviruses use the homotrimeric spike glycoprotein (comprising a S1 subunit and S2 subunit in each spike monomer) on the envelope to bind to their cellular receptors. Such binding triggers a cascade of events that leads to the fusion between cell and viral membranes for cell entry. Previous cryo-electron microscopy studies of the SARS-CoV spike protein and its interaction with the cell receptor ACE2 have shown that receptor binding induces the dissociation of the S1 with ACE2, prompting the S2 to transit from a metastable pre-fusion to a more-stable post-fusion state that is essential for membrane fusion ${ }^{9-12}$. Therefore, binding to the ACE2 receptor is a critical initial step for SARS-CoV to enter into target cells. Recent studies also highlighted the important role of ACE2 in mediating entry of SARS-CoV-2 ${ }^{1,13-15}$. HeLa cells expressing ACE2 are susceptible to SARS-CoV-2 infection whereas those without ACE2 are not ${ }^{1}$. In vitro binding measurements also showed that the SARS-CoV-2 RBD binds to ACE2 with an affinity in the low nanomolar range, indicating that the $R B D$ is a key functional component within the S1 subunit that is responsible for binding of SARS-CoV-2 by ACE2 ${ }^{13,16}$.

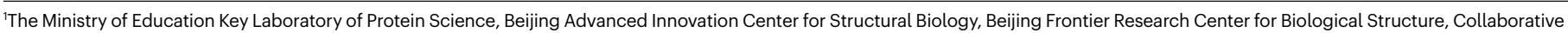

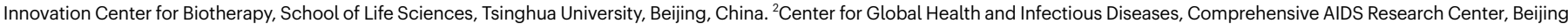

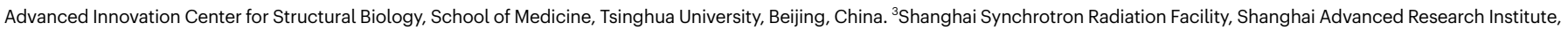

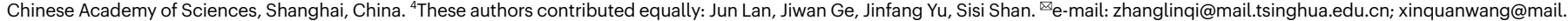
tsinghua.edu.cn 


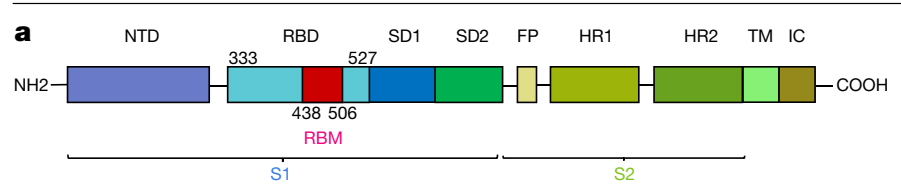

b
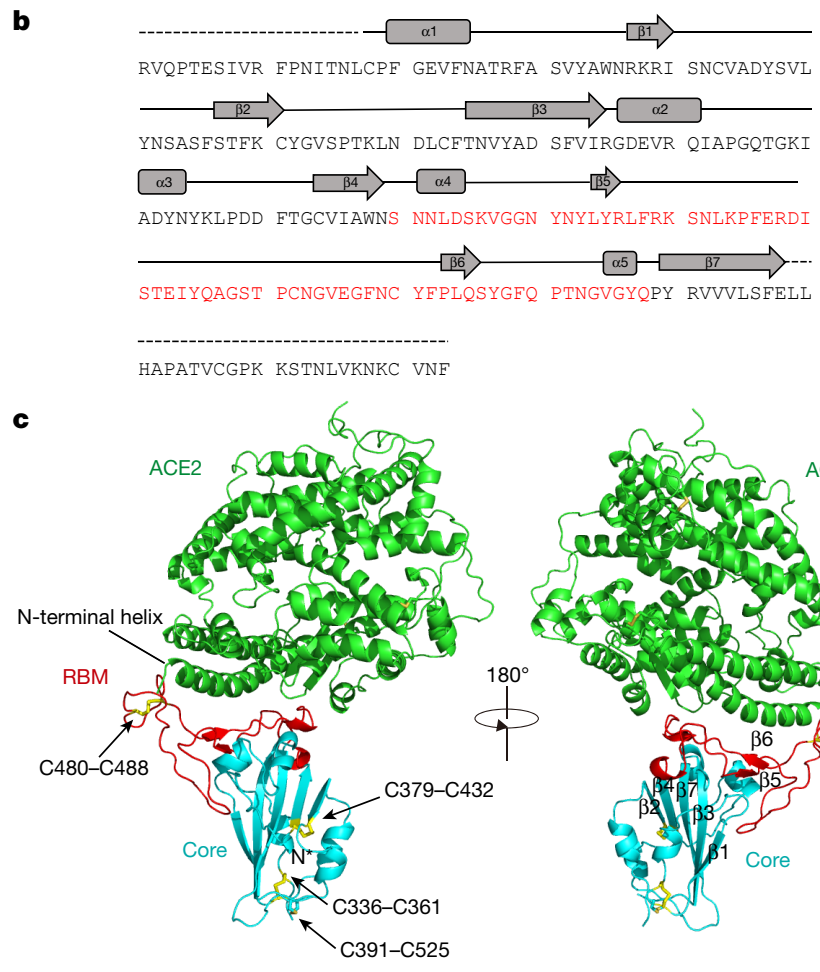

SARS-CoV-2 RBD

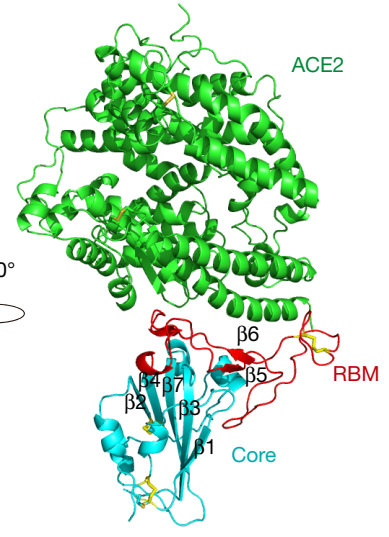

SARS-CoV-2 RBD

Fig. 1 | Overall structure of SARS-CoV-2 RBD bound to ACE2. a, Overall topology of the SARS-CoV-2 spike monomer. FP, fusion peptide; HR1, heptad repeat 1;HR2, heptad repeat 2; IC, intracellular domain; NTD, N-terminal domain; SD1, subdomain 1; SD2, subdomain 2; TM, transmembrane region. b, Sequence and secondary structures of SARS-CoV-2 RBD. The RBM sequence is shown in red.c, Overall structure of the SARS-CoV-2 RBD bound to ACE2. ACE2 is shown in green. The SARS-CoV-2 RBD core is shown in cyan and RBM in red. Disulfide bonds in the SARS-CoV-2 RBD are shown as sticks and indicated by arrows. The N-terminal helix of ACE2 responsible for binding is labelled.

The cryo-electron microscopy structure of the SARS-CoV-2 spike trimer has recently been reported in two independent studies ${ }^{13,17}$. However, inspection of one available spike structure revealed the incomplete modelling of the RBD, particularly for the receptor-binding motif (RBM) that interacts directly with ACE $2^{17}$. Computer modelling of the interaction between the SARS-CoV-2 RBD and ACE2 has identified some residues that are potentially involved in the interaction; however, the actual residues that mediate the interaction remained unclear ${ }^{18}$. Furthermore, despite detectable cross-reactive SARS-CoV-2-neutralizing activity of serum or plasma from patients who recovered from SARS-CoV infections ${ }^{15}$, no isolated SARS-CoV monoclonal antibodies are able to neutralize SARS-CoV-2 ${ }^{16,17}$. These findings highlight some of the intrinsic sequence and structure differences between the SARS-CoV and SARS-CoV-2 RBDs.

To elucidate the interaction between the SARS-CoV-2 RBD and ACE2 at a higher resolution, we determined the structure of the SARS-CoV-2 RBD-ACE2 complex using X-ray crystallography. This atomic-level structural information greatly improves our understanding of the interaction between SARS-CoV-2 and susceptible cells, provides a precise target for neutralizing antibodies, and assists the structure-based vaccine design that is urgently needed in the ongoing fight against SARS-CoV-2. Specifically, we expressed

the SARS-CoV-2 RBD (residues Arg319-Phe541) (Fig. 1a, b) and the $\mathrm{N}$-terminal peptidase domain of ACE2 (residues Ser19-Asp615) in $\mathrm{Hi} 5$ insect cells and purified them by Ni-NTA affinity purification and gel filtration (Extended Data Fig. 1). The structure of the complex was determined by molecular replacement using the SARS-CoV RBD and ACE2 structures as search models ${ }^{4}$, and refined to a resolution of $2.45 \AA$ with final $R_{\text {work }}$ and $R_{\text {free }}$ factors of $19.6 \%$ and $23.7 \%$, respectively (Extended Data Fig. 2 and Extended Data Table 1). The final model contains residues Thr333-Gly526 of the SARS-CoV-2 RBD, residues Ser19-Asp615 of the ACE2 N-terminal peptidase domain, one zinc ion, four $N$-acetyl- $\beta$-glucosaminide (NAG) glycans linked to ACE2 Asn90, Asn322 and Asn546 and to RBD Asn343, as well as 80 water molecules.

The SARS-CoV-2 RBD has a twisted five-stranded antiparallel $\beta$ sheet $(\beta 1, \beta 2, \beta 3, \beta 4$ and $\beta 7)$ with short connecting helices and loops that form the core (Fig. 1b, c). Between the $\beta 4$ and $\beta 7$ strands in the core, there is an extended insertion containing the short $\beta 5$ and $\beta 6$ strands, $\alpha 4$ and $\alpha 5$ helices and loops (Fig. 1b, c). This extended insertion is the RBM, which contains most of the contacting residues of SARS-CoV-2 that bind to ACE2. A total of nine cysteine residues are found in the RBD, eight of which form four pairs of disulfide bonds that are resolved in the final model. Among these four pairs, three are in the core (Cys336-Cys361, Cys379-Cys432 and Cys391-Cys525), which help to stabilize the $\beta$ sheet structure (Fig. 1c); the remaining pair (Cys480-Cys488) connects the loops in the distal end of the RBM (Fig. 1c). The N-terminal peptidase domain of ACE2 has two lobes, forming the peptide substrate binding site between them. The extended RBM in the SARS-CoV-2 RBD contacts the bottom side of the small lobe of ACE2, with a concave outer surface in the RBM that accommodates the N-terminal helix of the ACE2 (Fig. 1c). The overall structure of the SARS-CoV-2 RBD is similar to that of the SARS-CoV RBD (Extended Data Fig. 3a), with a root mean square deviation (r.m.s.d.) of $1.2 \AA$ for 174 aligned $C_{\alpha}$ atoms. Even in the RBM, which has more sequence variation, the overall structure is also highly similar (r.m.s.d. of $1.3 \AA$ ) to the SARS-CoV RBD, with only one obvious conformational change in the distal end (Extended Data Fig. 3a). The overall binding mode of the SARS-CoV-2 RBD to ACE2 is also nearly identical to that observed in the previously determined structure of the SARS-CoV RBD-ACE2 complex ${ }^{4}$ (Extended Data Fig. 3b).

The cradling of the $\mathrm{N}$-terminal helix of ACE2 by the outer surface of the RBM results in a large buried surface of $1,687 \AA^{2}\left(864 \AA^{2}\right.$ on the RBD and $823 \AA^{2}$ on the ACE2) at the SARS-CoV-2 RBD-ACE2 interface. A highly similar buried surface of $1,699 \AA^{2}$ contributed by SARS-CoV $\operatorname{RBD}\left(869 \AA^{2}\right)$ and ACE2 $\left(830 \AA^{2}\right)$ is also observed at the SARS-CoV RBDACE2 interface. With a distance cut-off of $4 \AA$, a total of 17 residues of the RBD are in contact with 20 residues of ACE2 (Fig. 2a and Extended Data Table 2). Analysis of the interface between the SARS-CoV RBD and ACE2 revealed a total of 16 residues of the SARS-CoV RBD in contact with 20 residues of ACE2 (Fig. 2a and Extended Data Table 2). Among the 20 ACE2 residues that interact with the two different RBDs, 17 residues are shared between both interactions and most of the contacting residues are located at the $\mathrm{N}$-terminal helix (Fig. 2a and Extended Data Table 2).

To compare the ACE2-interacting residues on the SARS-CoV-2 and SARS-CoV RBDs, we used structure-guided sequence alignment and mapped them to their respective sequences (Fig. 2b). Among 14 shared amino acid positions used by both RBMs for the interaction with ACE2, 8 have the identical residues between the two RBDs, including Tyr449/Tyr436, Tyr453/Tyr440, Asn487/ Asn473, Tyr489/Tyr475, Gly496/Gly482, Thr500/Thr486, Gly502/ Gly 488 and Tyr505/Tyr491 of SARS-CoV-2/SARS-CoV, respectively (Fig. 2b). Five positions have residues that have similar biochemical properties despite of having different side chains, including Leu455/Tyr442, Phe456/Leu443, Phe486/Leu472, GIn493/Asn479 and Asn501/Thr487 of SARS-CoV-2/SARS-CoV, respectively (Fig. 2b). 

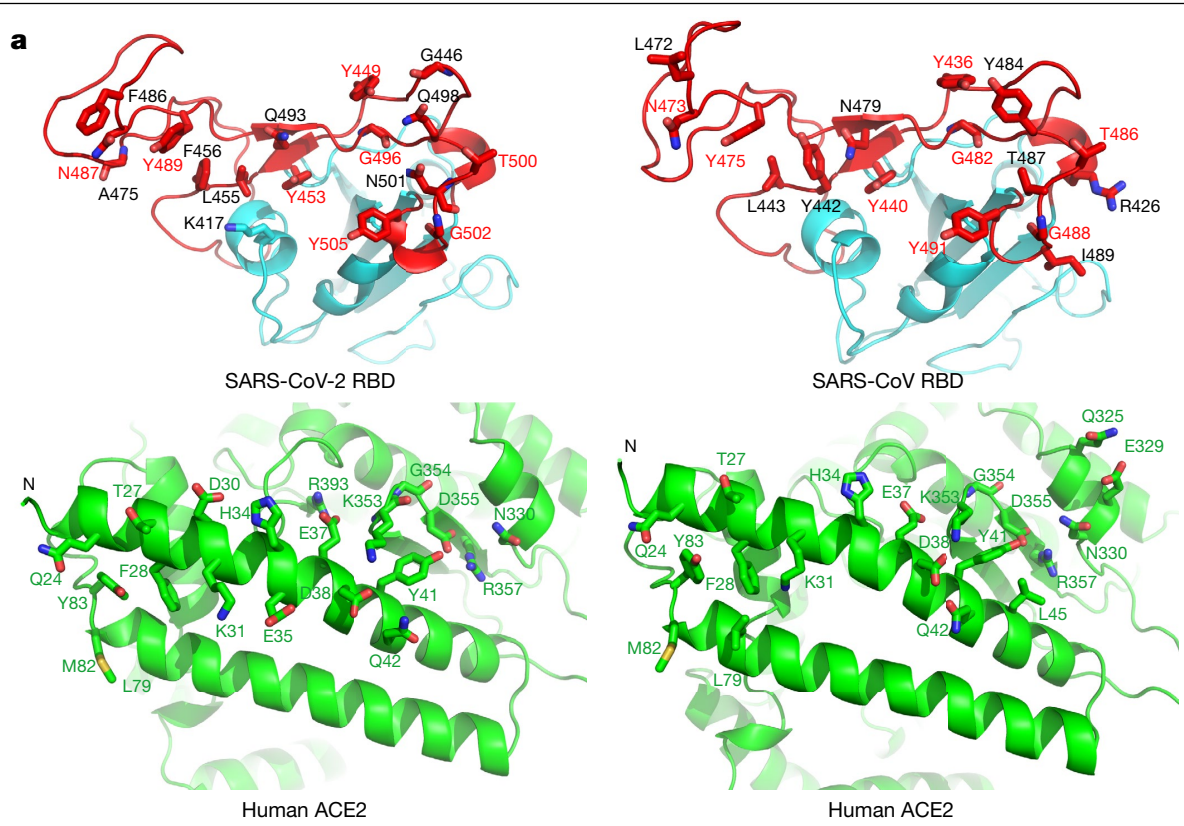

b

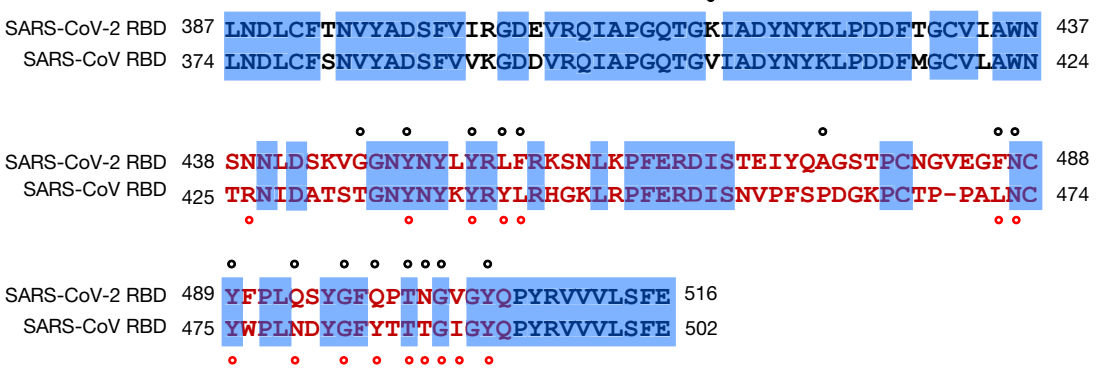

Fig. 2 | The SARS-CoV-2 RBD-ACE2 and SARS-CoV RBD-ACE2 interfaces. a, Contacting residues are shown as sticks at the SARS-CoV-2 RBD-ACE2 and SARS-CoV RBD-ACE2 interfaces. Positions in both RBDs that are involved in ACE2 binding are indicated by red labels. b, Sequence alignment of the

The remaining position is at the Gln498/Tyr484 location (Fig. 2b), at which GIn498 of SARS-CoV-2 and Tyr484 of SARS-CoV both interact with Asp38, Tyr41, Gln42, Leu45 and Lys353 of ACE2. Among the six RBD positions with changed residues, SARS-CoV residues Tyr442, Leu472, Asn 479 and Thr487 have previously been shown to be essential for binding ACE $2^{18}$. At the Leu455/Tyr442 position, Leu 455 of SARS-CoV-2 and Tyr442 of SARS-CoV have similar interactions with Asp30, Lys31 and His34 of ACE2 (Fig. 3a). At the Phe486/ Leu472 position, Phe486 of SARS-CoV-2 interacts with Gln24, Leu79, Met82 and Tyr83 of ACE2, whereas Leu472 of SARS-CoV has less interactions with Leu79 and Met82 of ACE2 (Fig. 3a). At the GIn493/ Asn479 position, Gln493 of SARS-CoV-2 interacts with Lys31, His34 and Glu35 of ACE2 and forms a hydrogen bond with Glu35; Asn479 of SARS-CoV interacts with only His34 of ACE2 (Fig. 3a). At the Asn501/ Thr487 position, both residues have similar interactions with Tyr41, Lys353, Gly354 and Asp355 of ACE2 (Fig. 3a). Asn501 of SARS-CoV-2 and Thr487 of SARS-CoV both form a hydrogen bond with Tyr41 of ACE2 (Fig. 3a). Outside the RBM, there is a unique ACE2-interacting residue (Lys417) in SARS-CoV-2, which forms salt-bridge interactions with Asp30 of ACE2 (Fig. 3b). This position is replaced by a valine in the SARS-CoV RBD that fails to participate in ACE2 binding (Figs. 2b, $3 b)$. Furthermore, a comparison of the surface electrostatic potential also identified a positive charged patch on the SARS-CoV-2 RBD contributed by Lys 417 that is absent on the SARS-CoV RBD (Fig. 3b). These subtly different ACE2 interactions may contribute to the difference in binding affinity of the SARS-CoV-2 and SARS-CoV
SARS-CoV-2 and SARS-CoV RBDs. Contacting residues in the SARS-CoV-2 RBD are indicated by black dots; contacting residues in the SARS-CoV RBD are indicated by red dots.

to the ACE2 receptor ( $4.7 \mathrm{nM}$ compared with $31 \mathrm{nM}$, respectively) (Extended Data Fig. 4).

One notable and common feature that was found for both RBDACE2 interfaces is the networks of hydrophilic interactions. There are 13 hydrogen bonds and 2 salt bridges at the SARS-CoV-2 RBD-ACE2 interface, and 13 hydrogen bonds and 3 salt bridges at the SARS-CoV RBD-ACE2 interface (Table1). The second shared feature is the involvement of multiple tyrosine residues that form hydrogen-bonding interactions with the polar hydroxyl group. These include Tyr449, Tyr489 and Tyr505 from the SARS-CoV-2 RBD and Tyr436, Tyr475 and Tyr491 from the SARS-CoV RBD (Table 1). The third shared feature may reside in the Asn90-linked glycans of the ACE2 that bind to different RBDs. In the structure of the SARS-CoV RBD-ACE2 complex, a chain of Asn90-linked NAG-NAG- $\beta$-D-mannose is in contact with Thr402 of the SARS-CoV RBD (Extended Data Fig. 5a), and this glycan-RBD interaction has been proposed to have important roles in the binding of SARS-CoV RBD by ACE2 ${ }^{4,19}$. In the SARS-CoV-2 RBD-ACE2 structure, the density enabled only the modelling of the first NAG linked to ACE2 Asn90, and no interactions between this NAG and the SARS-CoV-2 RBD were observed (Extended Data Fig. 5b). However, this does not exclude that glycans after the first NAG may interact with the SARS-CoV-2 RBD and may have important roles in the binding of SARS-CoV-2 RBD by ACE2. Taken together, our results show that the SARS-CoV-2 RBD-ACE2 and SARS-CoV RBD-ACE2 interfaces share substantial similarity in the buried surface area, the number of interacting residues and hydrophilic interaction networks, although 

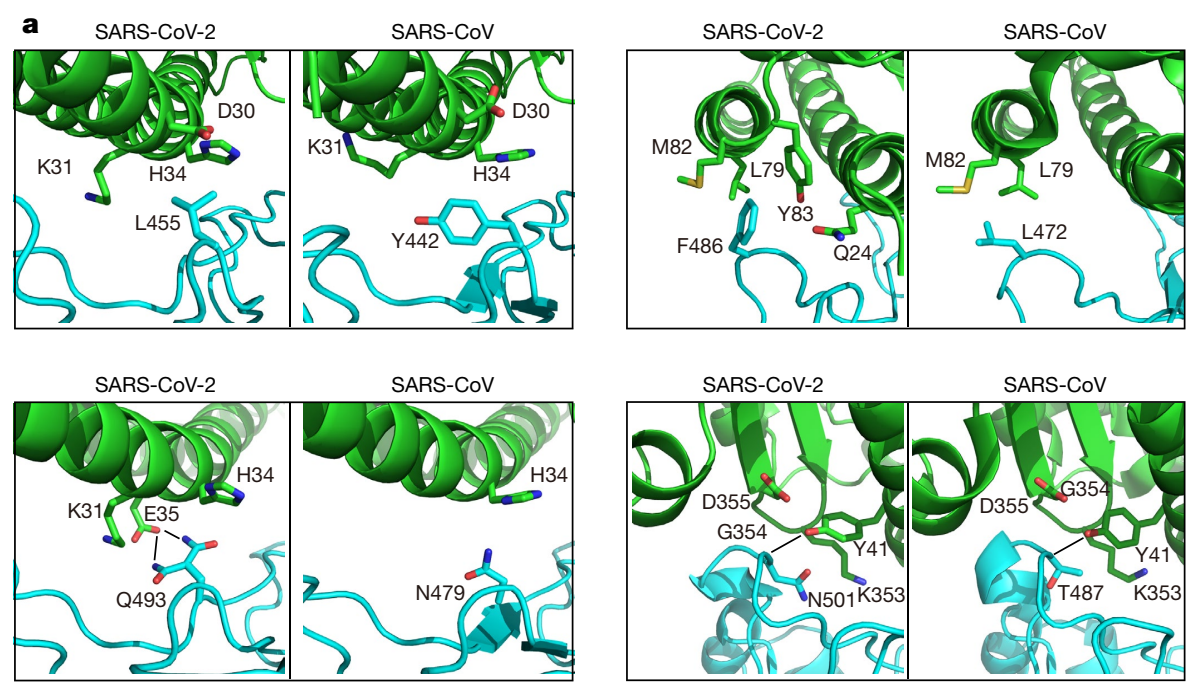

b
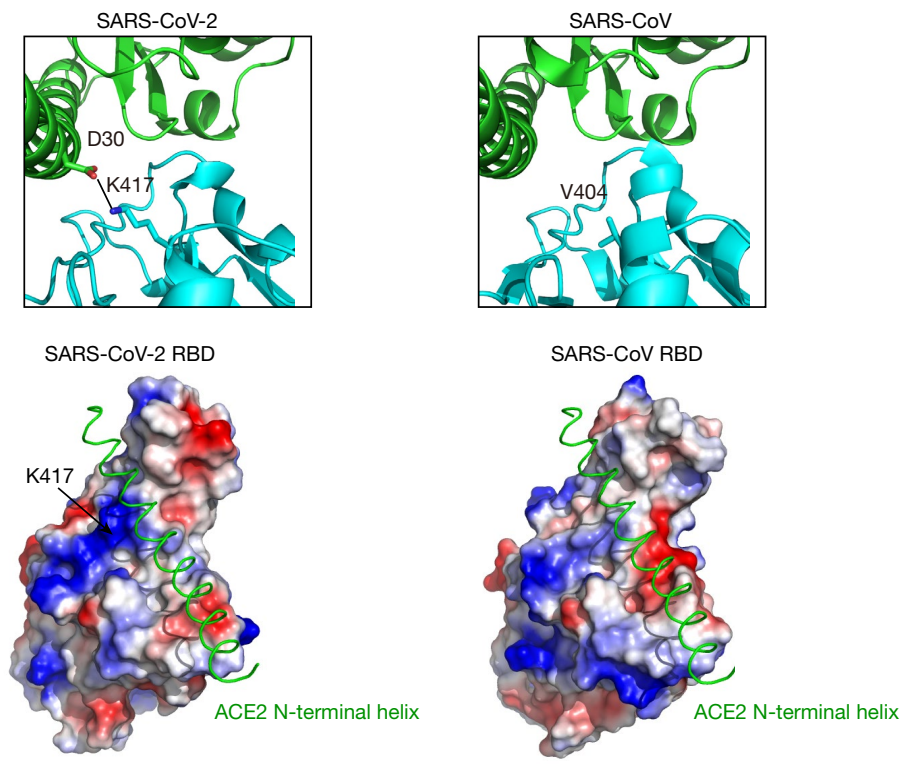

Fig. 3 | Comparisons of interactions at the SARS-CoV-2 RBD-ACE2 and SARS-COV RBD-ACE2 interfaces. $a$, Interactions around the SARS-CoV-2 and SARS-CoV positions in the RBM with changed residues. SARS-CoV-2 and SARS-CoV RBDs are shown in cyan. ACE2 is shown in green. $\mathbf{b}$, Interactions around the K417 and V404 positions of SARS-CoV-2 and SARS-CoV RBDs,

some of the ACE2 interactions observed both inside and outside the RBM were different (Fig. 3a, b). Such similarities argue strongly for the convergent evolution of the SARS-CoV-2 and SARS-CoV RBD structures to improve binding affinity to the same ACE2 receptor, although SARS-CoV-2 does not cluster within SARS-CoV and SARSr-CoV in the Betacoronavirus genus.

Consistent with the high structural similarity, we found that the binding affinities between ACE2 and SARS-CoV-2 and SARS-CoV RBDs also fall into a similar range. Specifically, the equilibrium dissociation constant $\left(K_{\mathrm{D}}\right)$ of ACE2 and SARS-CoV-2 RBD is $4.7 \mathrm{nM}$, and of ACE2 and SARS-CoV RBD is $31 \mathrm{nM}$ (Extended Data Fig. 4). Similar results have also been reported by other groups ${ }^{13,16}$. However, this is slightly different from a recent report in which an approximately 20 -fold increased binding between ACE2 and the SARS-CoV-2 spike trimer was found $\left(K_{\mathrm{D}}\right.$ of $14.7 \mathrm{nM}$ ) compared with that between ACE2 and SARS-CoV RBD-SD1 $\left(K_{\mathrm{D}} \text { of } 325 \mathrm{nM}\right)^{17}$. This is perhaps due to the different proteins used in the assay or because of other unknown reasons. Nevertheless, the binding affinity alone is unlikely to explain the unusual transmissibility of SARS-CoV-2. Other factors such as the unique 'RRAR' furin cleavage

respectively, that are outside the RBM and electrostatic potential maps of the SARS-CoV-2 and SARS-CoV RBDs. The position of K417 in the SARS-CoV-2 RBD is indicated by a black arrow. The $\mathrm{N}$-terminal helix of ACE2 is shown as a green ribbon. The Protein Data Bank (PDB) code for the SARS-CoV RBD-ACE2 complex is $2 \mathrm{AJF}$.

site at the S1-S2 boundary of the SARS-CoV-2 spike protein may have more-important roles in facilitating the rapid human-to-human transmission of SARS-CoV-2.

Neutralizing antibodies represent an important component of the immune system in the fight against viral infections. It has been reported that SARS-CoV-2 could be cross-neutralized by horse anti-SARS-CoV serum and convalescent serum from a patient with a SARS-CoV infection ${ }^{1,15}$, reinforcing the structural similarity between the RBDs of SARS-CoV-2 and SARS-CoV. Such similarity also increased the hope of the rapid application of previously characterized SARS-CoV monoclonal antibodies in the clinical setting. However, no antibody that targeted SARS-CoV (m396, S230, 80R and CR3014) has so far demonstrated any notable cross-binding and neutralization activity against spike protein or RBD of SARS-CoV-2 ${ }^{16,17,20-23}$. One exception is SARS-CoV antibody CR3022 that binds to the SARS-CoV-2 RBD with a $K_{\mathrm{D}}$ of $6.2 \mathrm{nM}$, although its neutralizing activity against SARS-CoV-2 has not yet been reported ${ }^{16}$. Currently, we are uncertain where exactly the epitope of CR3022 on the RBDs of SARS-CoV or SARS-CoV-2 is located. Among the three antibodies that are incapable 
Table 1 | The hydrogen bonds and salt bridges at the SARS-CoV-2 RBD-ACE2 and SARS-CoV RBD-ACE2 interfaces

\begin{tabular}{|c|c|c|c|c|c|}
\hline & SARS-CoV-2 RBD & Length (Å) & ACE2 & Length (Å) & SARS-CoV RBD \\
\hline \multirow[t]{19}{*}{ Hydrogen bonds } & N487(ND2) & 2.6 & Q24(OE1) & 2.9 & N473(ND2) \\
\hline & K417(NZ) & 3.0 & D30(OD2) & & \\
\hline & Q493(NE2) & 2.8 & E35(OE2) & & \\
\hline & & & E37(OE1) & 3.4 & Y491(OH) \\
\hline & $\mathrm{Y} 505(\mathrm{OH})$ & 3.2 & E37(OE2) & & \\
\hline & & & D38(OD1) & 3.0 & Y436(OH) \\
\hline & $\mathrm{Y} 449(\mathrm{OH})$ & 2.7 & D38(OD2) & 3.0 & $\mathrm{Y} 436(\mathrm{OH})$ \\
\hline & T500(OG1) & 2.6 & Y41(OH) & 2.8 & T486(OG1) \\
\hline & $\mathrm{N} 501(\mathrm{~N})$ & 3.7 & Y41(OH) & 3.3 & T487(N) \\
\hline & G446(O) & 3.3 & Q42(NE2) & & \\
\hline & $\mathrm{Y} 449(\mathrm{OH})$ & 3.0 & Q42(NE2) & & \\
\hline & & & Q42(OE1) & 2.7 & $\mathrm{Y} 436(\mathrm{OH})$ \\
\hline & $\mathrm{Y} 489(\mathrm{OH})$ & 3.5 & Y83(OH) & 3.3 & Y475(OH) \\
\hline & N487(OD1) & 2.7 & $\mathrm{Y} 83(\mathrm{OH})$ & 2.8 & N473(ND2) \\
\hline & & & Q325(OE1) & 3.8 & $\mathrm{R} 426(\mathrm{NH} 2)$ \\
\hline & & & E329(OE2) & 3.0 & $\mathrm{R} 426(\mathrm{NH} 2)$ \\
\hline & & & N330(ND2) & 2.8 & $\mathrm{~T} 486(\mathrm{O})$ \\
\hline & G502(N) & 2.8 & $\mathrm{~K} 353(\mathrm{O})$ & 2.6 & G488(N) \\
\hline & Y505(OH) & 3.7 & R393(NH2) & & \\
\hline \multirow[t]{5}{*}{ Salt bridges } & K417(NZ) & 3.9 & D30(OD1) & & \\
\hline & K417(NZ) & 3.0 & D30(OD2) & & \\
\hline & & & E329(OE2) & 3.7 & $\mathrm{R} 426(\mathrm{NH} 1)$ \\
\hline & & & E329(OE1) & 3.9 & $\mathrm{R} 426(\mathrm{NH} 2)$ \\
\hline & & & E329(OE2) & 3.0 & $\mathrm{R} 426(\mathrm{NH} 2)$ \\
\hline
\end{tabular}

$\mathrm{ND2}$, nitrogen delta 2; NE2, nitrogen epsilon 2; $\mathrm{NZ}$, nitrogen zeta; $\mathrm{N}$, nitrogen; $\mathrm{NH1}$, nitrogen eta 1: $\mathrm{NH} 2$, nitrogen eta 2; $\mathrm{OH}$, oxygen eta; $\mathrm{O}$, oxygen; $\mathrm{OD1}$, oxygen delta 1; $\mathrm{OD2}$, oxygen delta 2; $\mathrm{OG1}$, oxygen gamma 1; OE1, oxygen epsilon 1; OE2, oxygen epsilon 2.

of binding to the SARS-CoV-2 RBD, two (m396 and 80R) have their epitopes resolved by the high-resolution crystal-structure determination of SARS-CoV RBD-Fab complexes ${ }^{20,21}$. By mapping these epitope residues onto the sequence of SARS-CoV RBD aligned with the sequence of SARS-CoV-2 RBD (Fig. 4), we found that antibody $\mathrm{m} 396$ has 7 residue changes in the SARS-CoV-2 RBD among 21 epitope positions (Fig. 4). There are 16 residue changes in the SARS-CoV-2 RBD among 25 epitope positions of antibody 80R (Fig. 4). This may provide a structural basis for the lack of cross-reactivity of m396 and $80 \mathrm{R}$ with SARS-CoV-2. The cross-neutralization of SARS-CoV-2 by horse anti-SARS-CoV serum and serum or plasma from patients recovered from SARS-CoV infections reveals a great potential in

SARS-CoV-2 RBD 336 CPFGEVFNATRFASVYAWNRKRI SNCVADYSVLYNSASFSTFKCYGVSPTK 386 SARS-COV RBD 323 CPFGEVFNATKFPSVYAWERKKI SNCVADYSVLYNSTFFSTFKCYGVSATK 373

SARS-COV-2 RBD 387 LNDLCETNVYADSFVIRGDEVRQIAPGQTGKIADYNYKLPDDFTGCVIAWN 437 SARS-COV RBD 374 LNDLCFSNVYADSFVVKGDDVRQIAPGQTGVIADYNYKLPDDFMGCVLAWN 424

SARS-CoV-2 RBD 438 SNNLDSKVGGNYNYLYRLFRKSNLKPFERDISTEIYQAGSTPCNGVEGFNC 488 SARS-COV RBD 425 TRNIDATSTGNYNYKYRYLRHGKLRPFERDISNVPFSPDGKPCTP-PALNC 474 : $0: 0$ ० ०.。

SARS-CoV-2 RBD 489 YFPLQSYGEQPTNGVGYQPYRVVVLSFE 516

SARS-CoV RBD 475 YWPLNDYGFYTTTGIGYQPYRVVVLSFE $502 \quad \bullet m 396$ •80R

Fig. 4 | Mapping of SARS-CoV neutralizing antibody epitopes. The epitopes of SARS-CoV neutralizing antibodies $\mathrm{m} 396$ and $80 \mathrm{R}$, which target the RBD, are labelled in the SARS-CoV sequence aligned with the sequence of SARS-CoV-2 RBD. Epitope residues of $\mathrm{m} 396$ are indicated by black dots; epitope residues of $80 \mathrm{R}$ are indicated by red dots. identifying antibodies with cross-reactivity between these two coronaviruses $^{1,15}$. The conserved non-RBD regions in the spike protein, such as the S2 subunit, are the potential targets for cross-reactive antibodies. Although the RBD is less conserved, identical residues between SARS-CoV-2 and SARS-CoV RBD exist, even in the more variable RBM (Fig. 4). Considering that the RBD is the important region for receptor binding, antibodies that target the conserved epitopes in the RBD will also present a great potential for developing highly potent cross-reactive therapeutic agents against diverse coronavirus species, including SARS-CoV-2.

\section{Online content}

Any methods, additional references, Nature Research reporting summaries, source data, extended data, supplementary information, acknowledgements, peer review information; details of author contributions and competing interests; and statements of data and code availability are available at https://doi.org/10.1038/s41586-020-2180-5.

1. Zhou, P. et al. A pneumonia outbreak associated with a new coronavirus of probable bat origin. Nature 579, 270-273 (2020).

2. Wu, F. et al. A new coronavirus associated with human respiratory disease in China. Nature 579, 265-269 (2020).

3. Zhu, N. et al. A novel coronavirus from patients with pneumonia in China, 2019. N. Engl. J. Med. 382, 727-733 (2020).

4. Li, F., Li, W., Farzan, M. \& Harrison, S. C. Structure of SARS coronavirus spike receptor-binding domain complexed with receptor. Science 309, 1864-1868 (2005).

5. Lu, R. et al. Genomic characterisation and epidemiology of 2019 novel coronavirus: implications for virus origins and receptor binding. Lancet 395, 565-574 (2020).

6. Huang, C. et al. Clinical features of patients infected with 2019 novel coronavirus in Wuhan, China. Lancet 395, 497-506 (2020).

7. Liu, K. et al. Clinical characteristics of novel coronavirus cases in tertiary hospitals in Hubei Province. Chin. Med. J. (Engl.) https://doi.org/10.1097/CM9.0000000000000744 (2020). 


\section{Article}

8. Wang, D. et al. Clinical characteristics of 138 hospitalized patients with 2019 nove coronavirus-infected pneumonia in Wuhan, China. J. Am. Med. Assoc. 323, 1061-1069 (2020).

9. Gui, M. et al. Cryo-electron microscopy structures of the SARS-CoV spike glycoprotein reveal a prerequisite conformational state for receptor binding. Cell Res. 27, 119-129 (2017).

10. Song, W., Gui, M., Wang, X. \& Xiang, Y. Cryo-EM structure of the SARS coronavirus spike glycoprotein in complex with its host cell receptor ACE2. PLoS Pathog. 14, e1007236 (2018)

11. Kirchdoerfer, R. N. et al. Stabilized coronavirus spikes are resistant to conformational changes induced by receptor recognition or proteolysis. Sci. Rep. 8, 15701 (2018).

12. Yuan, Y. et al. Cryo-EM structures of MERS-CoV and SARS-CoV spike glycoproteins reveal the dynamic receptor binding domains. Nat. Commun. 8, 15092 (2017).

13. Walls, A. C. et al. Structure, function, and antigenicity of the SARS-CoV-2 spike glycoprotein. Cell https://doi.org/10.1016/j.cell.2020.02.058 (2020).

14. Letko, M., Marzi, A. \& Munster, V. Functional assessment of cell entry and receptor usage for SARS-CoV-2 and other lineage B betacoronaviruses. Nat. Microbiol. 5, 562-569 (2020)

15. Hoffmann, M. et al. SARS-CoV-2 cell entry depends on ACE2 and TMPRSS2 and is blocked by a clinically proven protease inhibitor. Cell https://doi.org/10.1016/j.cell.2020.02.052 (2020)

16. Tian, X. et al. Potent binding of 2019 novel coronavirus spike protein by a SARS coronavirus-specific human monoclonal antibody. Emerg. Microbes Infect. 9, 382-385 (2020)
17. Wrapp, D. et al. Cryo-EM structure of the 2019-nCoV spike in the prefusion conformation. Science 367, 1260-1263 (2020).

18. Wan, Y., Shang, J., Graham, R., Baric, R. S. \& Li, F. Receptor recognition by nove coronavirus from Wuhan: an analysis based on decade-long structural studies of SARS. J. Virol. 94, e00127-20 (2020).

19. Li, W. et al. Receptor and viral determinants of SARS-coronavirus adaptation to human ACE2. EMBO J. 24, 1634-1643 (2005)

20. Prabakaran, P. et al. Structure of severe acute respiratory syndrome coronavirus receptor-binding domain complexed with neutralizing antibody. J. Biol. Chem. $\mathbf{2 8 1}$ 15829-15836 (2006).

21. Hwang, W. C. et al. Structural basis of neutralization by a human anti-severe acute respiratory syndrome spike protein antibody, 8OR. J. Biol. Chem. 281, 34610-34616 (2006).

22. Walls, A. C. et al. Unexpected receptor functional mimicry elucidates activation of coronavirus fusion. Cell 176, 1026-1039 (2019).

23. van den Brink, E. N. et al. Molecular and biological characterization of human monoclonal antibodies binding to the spike and nucleocapsid proteins of severe acute respiratory syndrome coronavirus. J. Virol. 79, 1635-1644 (2005).

Publisher's note Springer Nature remains neutral with regard to jurisdictional claims in published maps and institutional affiliations.

(c) The Author(s), under exclusive licence to Springer Nature Limited 2020 


\section{Methods}

\section{Data reporting}

No statistical methods were used to predetermine sample size. The experiments were not randomized and the investigators were not blinded to allocation during experiments and outcome assessment.

\section{Protein expression and purification}

The SARS-CoV-2 RBD and the N-terminal peptidase domain of human ACE2 were expressed using the Bac-to-Bac baculovirus system (Invitrogen). The SARS-CoV-2RBD (residues Arg319-Phe541) with an N-terminal gp67 signal peptide for secretion and a C-terminal $6 \times$ His tag for purification was inserted into the pFastBac-Dual vector (Invitrogen). The construct was transformed into bacterial DH10Bac competent cells, and the extracted bacmid was then transfected into Sf9 cells using Cellfectin II Reagent (Invitrogen). The low-titre viruses were collected and then amplified to generate high-titre virus stocks, which were used to infect $\mathrm{Hi} 5$ cells at a density of $2 \times 10^{6}$ cells per $\mathrm{ml}$. The supernatant of cell culture containing the secreted SARS-CoV-2 RBD was collected $60 \mathrm{~h}$ after infection, concentrated and buffer-exchanged to HBS (10 mM HEPES, $\mathrm{pH} 7.2,150 \mathrm{mM} \mathrm{NaCl}$ ). The SARS-CoV-2 RBD was captured by Ni-NTA resin (GE Healthcare) and eluted with $500 \mathrm{mM}$ imidazole in HBS buffer. The SARS-CoV-2 RBD was then purified by gel filtration chromatography using a Superdex 200 column (GE Healthcare) pre-equilibrated with HBS buffer. Fractions containing the SARS-CoV-2 RBD were collected.

The N-terminal peptidase domain of human ACE2 (residues Ser19Asp615) was expressed and purified by essentially the same protocol as used for the SARS-CoV-2 RBD. To purify the SARS-CoV-2 RBD-ACE2 complex, ACE2 was incubated with the SARS-CoV-2 RBD for $1 \mathrm{~h}$ on ice in HBS buffer, and the mixture was then subjected to gel filtration chromatography. Fractions containing the complex were pooled and concentrated to $13 \mathrm{mg} \mathrm{ml}^{-1}$.

\section{Crystallization and data collection}

Crystals were successfully grown at room temperature in sitting drops, over wells containing $100 \mathrm{mM}$ MES, pH 6.5,10\% PEG 5000 MME and $12 \%$ 1-propanol. The drops were made by mixing $200 \mathrm{nl}$ of the SARS-CoV-2 RBD-ACE2 complex in $20 \mathrm{mM}$ Tris pH 7.5, $150 \mathrm{mM} \mathrm{NaCl}$ with $200 \mathrm{nl}$ well solution. Crystals were collected, soaked briefly in $100 \mathrm{mM}$ MES, pH 6.5, 10\% PEG 5000 MME, 12\% 1-propanol and 20\% glycerol, and were subsequently flash-frozen in liquid nitrogen. Diffraction data were collected at $100 \mathrm{~K}$ and at a wavelength of $1.07180 \AA$ A on the BL17U1 beam line of the Shanghai Synchrotron Research Facility. Diffraction data were autoprocessed using the aquarium pipeline ${ }^{24}$ and the data-processing statistics are listed in Extended Data Table 1.

\section{Structure determination and refinement}

The structure was determined using the molecular replacement method with PHASER in the CCP4 suite ${ }^{25}$. The search models used included the ACE2 extracellular domain and SARS-CoV RBD (PDB code 2AJF). Density map improvement by updating and refinement of the atoms was performed with $\mathrm{ARP} / \mathrm{WARP}^{26}$. Subsequent model building and refinement were performed using COOT and PHENIX, respectively ${ }^{27,28}$. Final Ramachandran statistics: $96.44 \%$ favoured, $3.56 \%$ allowed and $0.00 \%$ outliers for the final structure. The structure refinement statistics are listed in Extended Data Table 1. All structure figures were generated with $\mathrm{PyMol}^{29}$.

\section{Surface plasmon resonance experiments}

ACE2 was immobilized on a CM5 sensorchip (GE Healthcare) to a level of around 500 response units using a Biacore T200 (GE Healthcare) and a running buffer composed of $10 \mathrm{mM} \mathrm{HEPES} \mathrm{pH} \mathrm{7.2,150} \mathrm{mM} \mathrm{NaCl}$ and 0.05\% Tween-20. Serial dilutions of the SARS-CoV RBD and SARS-CoV-2 RBD were flowed through with a concentration ranging from 62.5 to $1.9 \mathrm{nM}$. The resulting data were fit to a $1: 1$ binding model using Biacore Evaluation Software (GE Healthcare).

\section{Reporting summary}

Further information on research design is available in the Nature Research Reporting Summary linked to this paper.

\section{Data availability}

The coordinates and structure factor files for the SARS-CoV-2 RBDACE2 complex have been deposited in the Protein Data Bank (PDB) under accession number $6 \mathrm{MOJ}$.

24. Yu, F. et al. Aquarium: an automatic data-processing and experiment information management system for biological macromolecular crystallography beamlines. J. Appl. Crystallogr. 52, 472-477 (2019).

25. McCoy, A. J. et al. Phaser crystallographic software. J. Appl. Crystallogr. 40, 658-674 (2007)

26. Cohen, S. X. et al. ARP/wARP and molecular replacement: the next generation. Acta Crystallogr. D 64, 49-60 (2008)

27. Emsley, P. \& Cowtan, K. Coot: model-building tools for molecular graphics. Acta Crystallogr. D 60, 2126-2132 (2004).

28. Adams, P. D. et al. PHENIX: building new software for automated crystallographic structure determination. Acta Crystallogr. D 58, 1948-1954 (2002).

29. Janson, G., Zhang, C.. Prado, M. G. \& Paiardini, A. PyMod 2.0: improvements in protein sequence-structure analysis and homology modeling within PyMOL. Bioinformatics $\mathbf{3 3}$ 444-446 (2017)

Acknowledgements We thank the staff at the BL17U1 beam line of the Shanghai Synchrotron Research Facility for data collection and processing. We thank staff at the X-ray crystallography platform of the Tsinghua University Technology Center for Protein Research for providing facility support. This work was supported by funds from the National Key Plan for Scientific Research and Development of China (grant number 2016YFD0500307). Research is also supported by the Tsinghua University Initiative Scientific Research Program (20201080053), National Natural Science Foundation Award (81530065), Beijing Municipal Science and Technology Commission (171100000517-001 and 171100000517-003), Tencent Foundation, Shuidi Foundation and TH Capital.

Author contributions J.L. and J.G. carried out protein expression, purification, crystallization, diffraction data collection and structure determination with the help of J.Y. and S.S. Q.Z. and X.S. helped with protein expression and purification. S.F., H.Z. and Q.W. helped with the collection of crystallization and diffraction data. X.W. and L.Z. conceived, designed and directed the study. J.L., J.G., J.Y., S.S., L.Z. and X.W. analysed the data, generated the figures and wrote the manuscript.

Competing interests The authors declare no competing interests.

Additional information

Supplementary information is available for this paper at https://doi.org/10.1038/s41586-020 $2180-5$.

Correspondence and requests for materials should be addressed to L.Z. or X.W. Peer review information Nature thanks Lijun Rong and the other, anonymous, reviewer(s) for their contribution to the peer review of this work. Peer reviewer reports are available. Reprints and permissions information is available at http://www.nature.com/reprints. 


\section{Article}

a

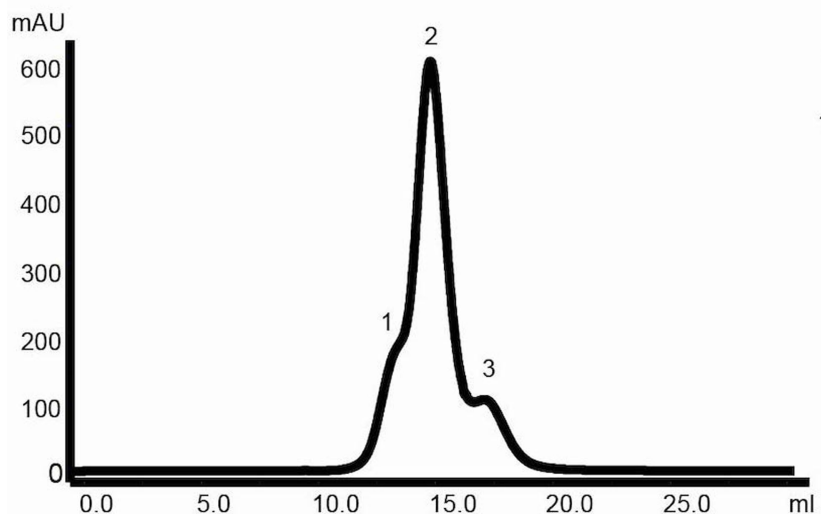

b
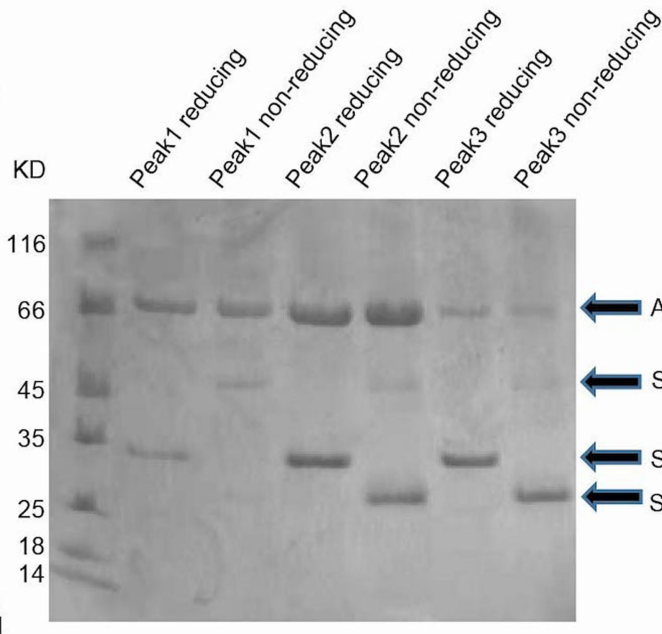

$\Longleftarrow$ ACE2

SARS-CoV-2 RBD dimer

$\Longleftarrow$ SARS-CoV-2 RBD monomer

SARS-CoV-2 RBD monomer

Extended Data Fig. 1 | Purification of the SARS-CoV-2 RBD-ACE2 complex. a, Gel filtration chromatography of the complex.1, SARS-CoV-2 RBD dimer-ACE2;2, SARS-CoV-2 RBD monomer-ACE2; 3, SARS-CoV-2 RBD monomer.b, SDS-PAGE gel of the complex. 

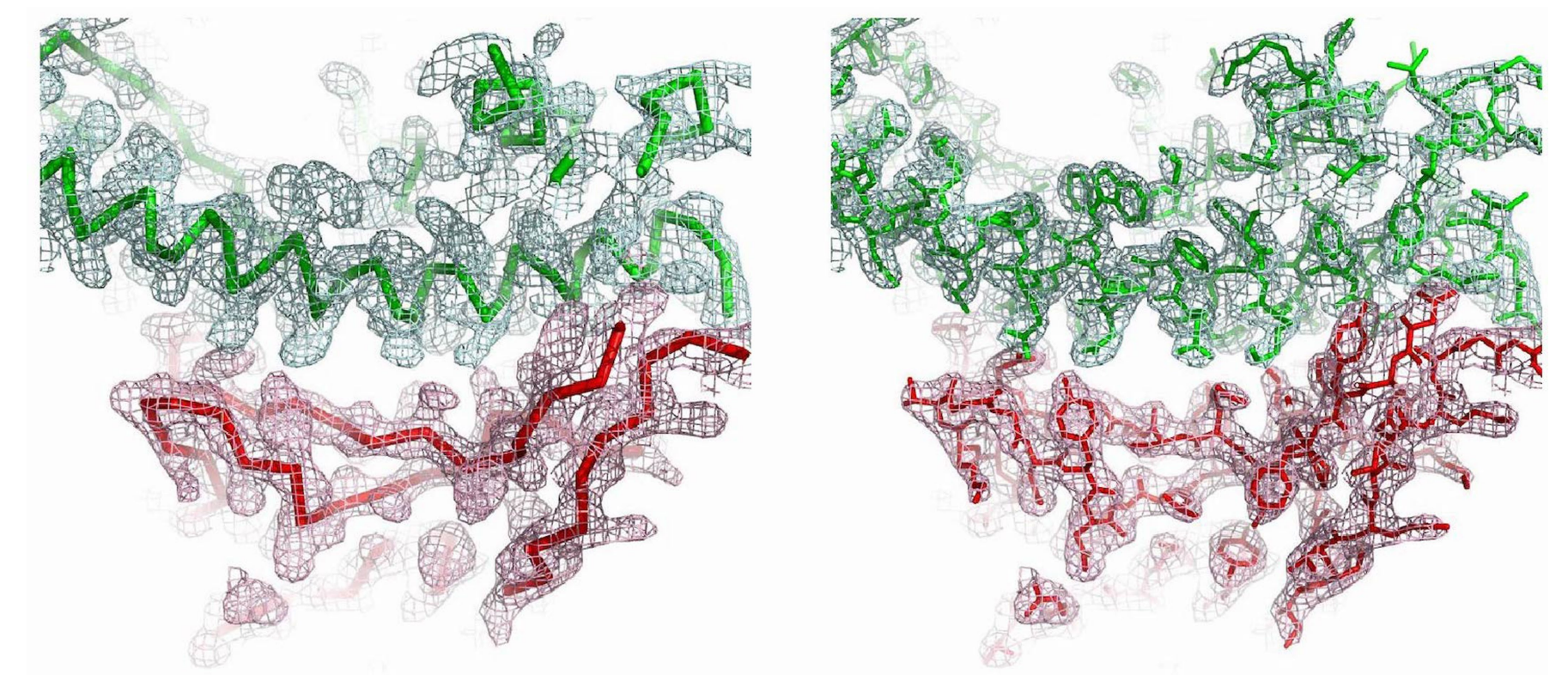

Extended Data Fig. 2 Electron density map. $2 F_{0}-F_{\mathrm{c}}$ electron density maps contoured at $1.5 \sigma$ at the binding interface between the SARS-CoV-2 RBD (red) and ACE2 (green) are shown. 


\section{Article}

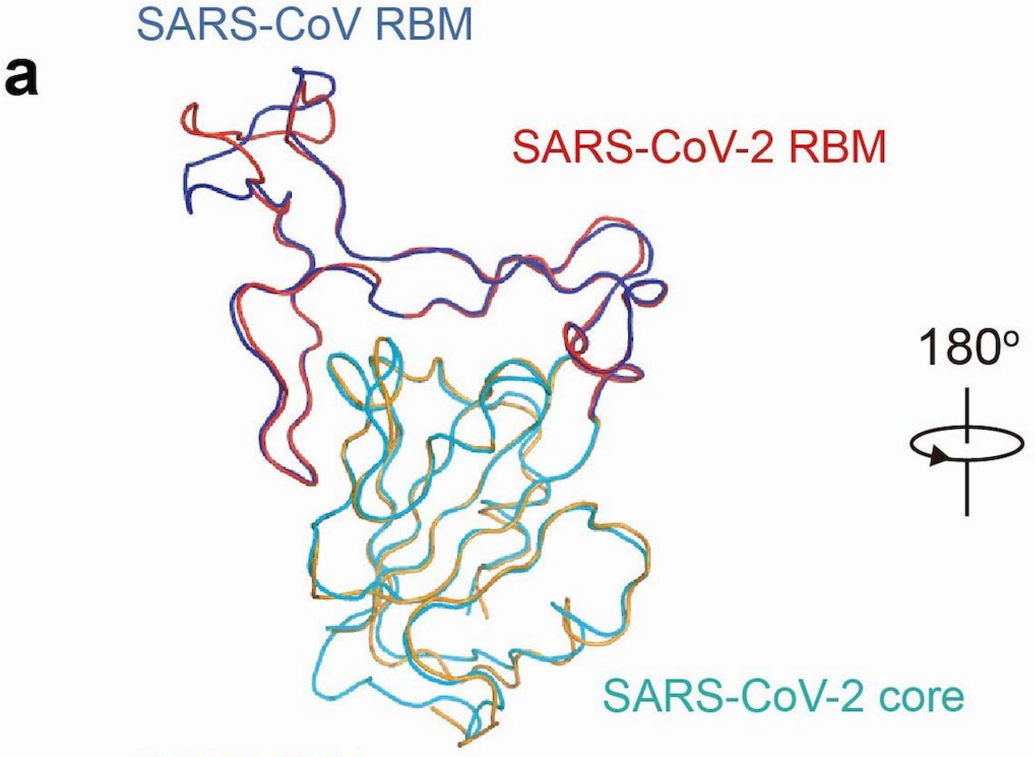

b

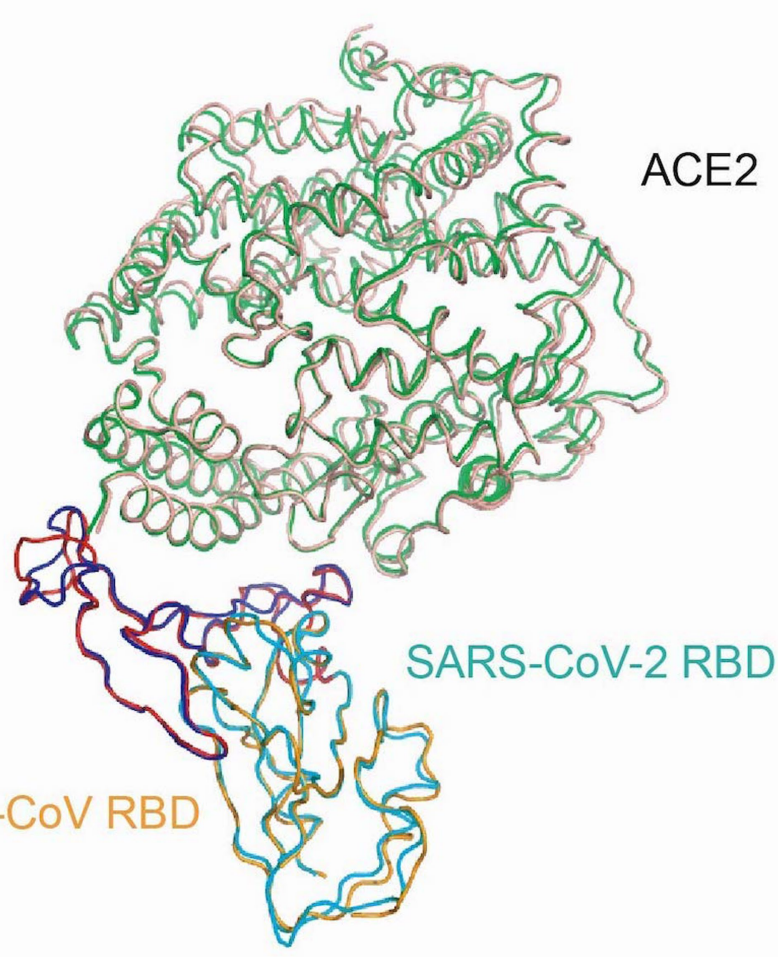

Extended Data Fig. 3 | Structural comparisons of the RBDs of SARS-CoV-2 and SARS-CoV and their binding modes to the ACE2 receptor. a, Alignment of the SARS-CoV-2RBD (core in cyan and RBM in red) and SARS-CoV RBD (core in orange and RBM in blue) structures. $\mathbf{b}$, Structural alignment of the


SARS-CoV-2 RBD-ACE2 and SARS-CoV RBD-ACE2 complexes. The SARS-CoV-2 RBD is shown in cyan and red, its interacting ACE2 is shown in green. The SARS-CoV RBD is shown in orange and blue, its interacting ACE2 is shown in salmon. The PDB code for the SARS-CoV RBD-ACE2 complex is 2AJF. 


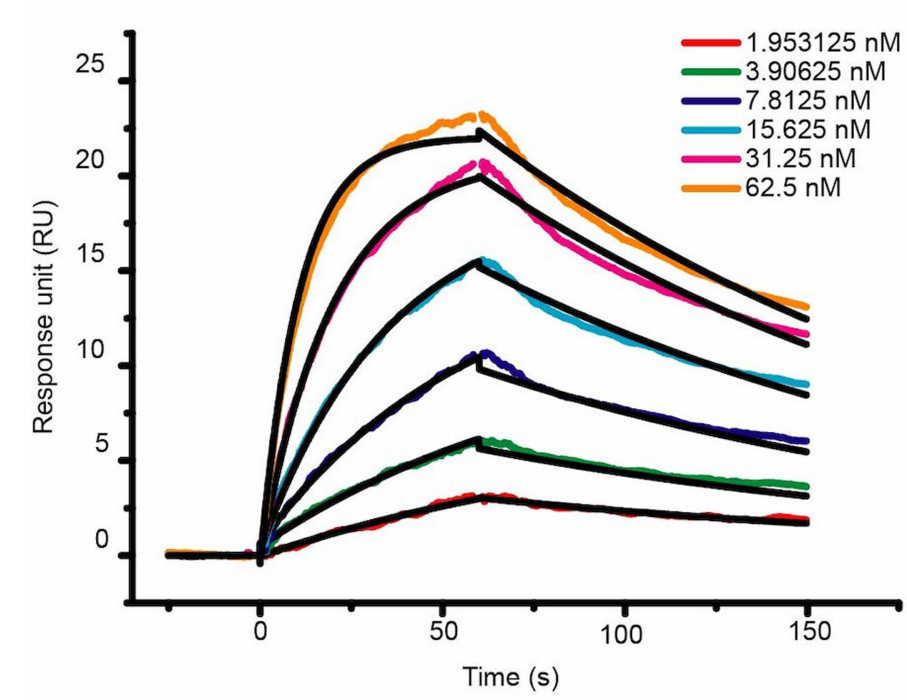

$k a=1.400 \times 10^{6}(1 / \mathrm{Ms})$

$\mathrm{kd}=6.544 \times 10^{-3}(1 / \mathrm{s})$

$\mathrm{K}_{\mathrm{D}}=4.674 \times 10^{-9} \mathrm{M}$

SARS-CoV-2

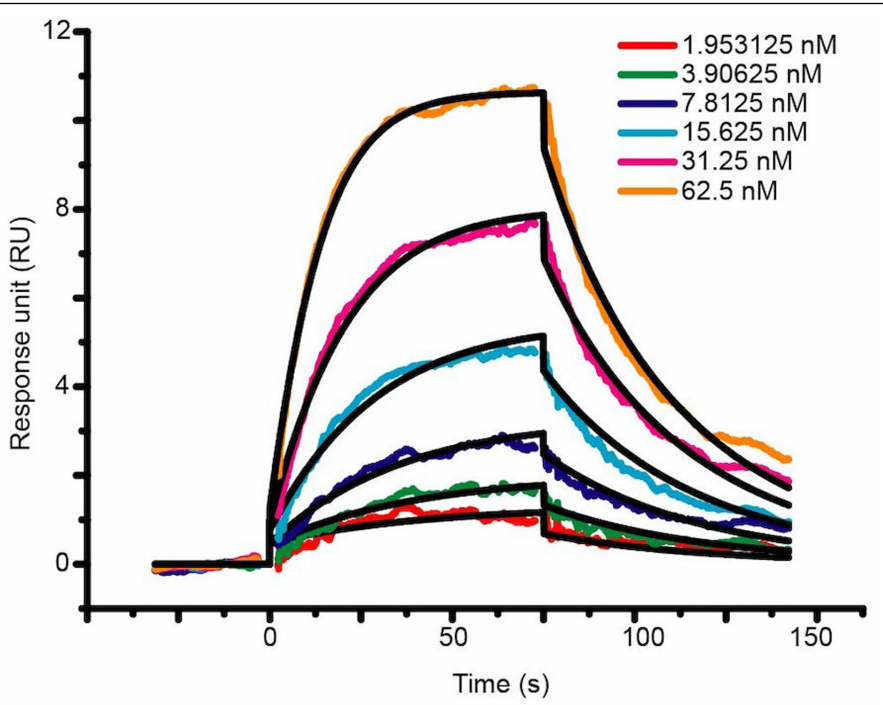

$k a=1.367 \times 10^{6}(1 / \mathrm{Ms})$

$\mathrm{kd}=4.317 \times 10^{-2}(1 / \mathrm{s})$

$\mathrm{K}_{\mathrm{D}}=3.159 \times 10^{-8} \mathrm{M}$

\section{SARS-CoV}

Extended Data Fig. 4 | Surface plasmon resonance sensorgrams. Binding curves of immobilized human ACE2 with the SARS-CoV-2 RBD (left) and SARS-CoV RBD (right). Data are shown as different coloured lines and the best fit of the data to a 1:1 binding model is shown in black. 


\section{Article}

a

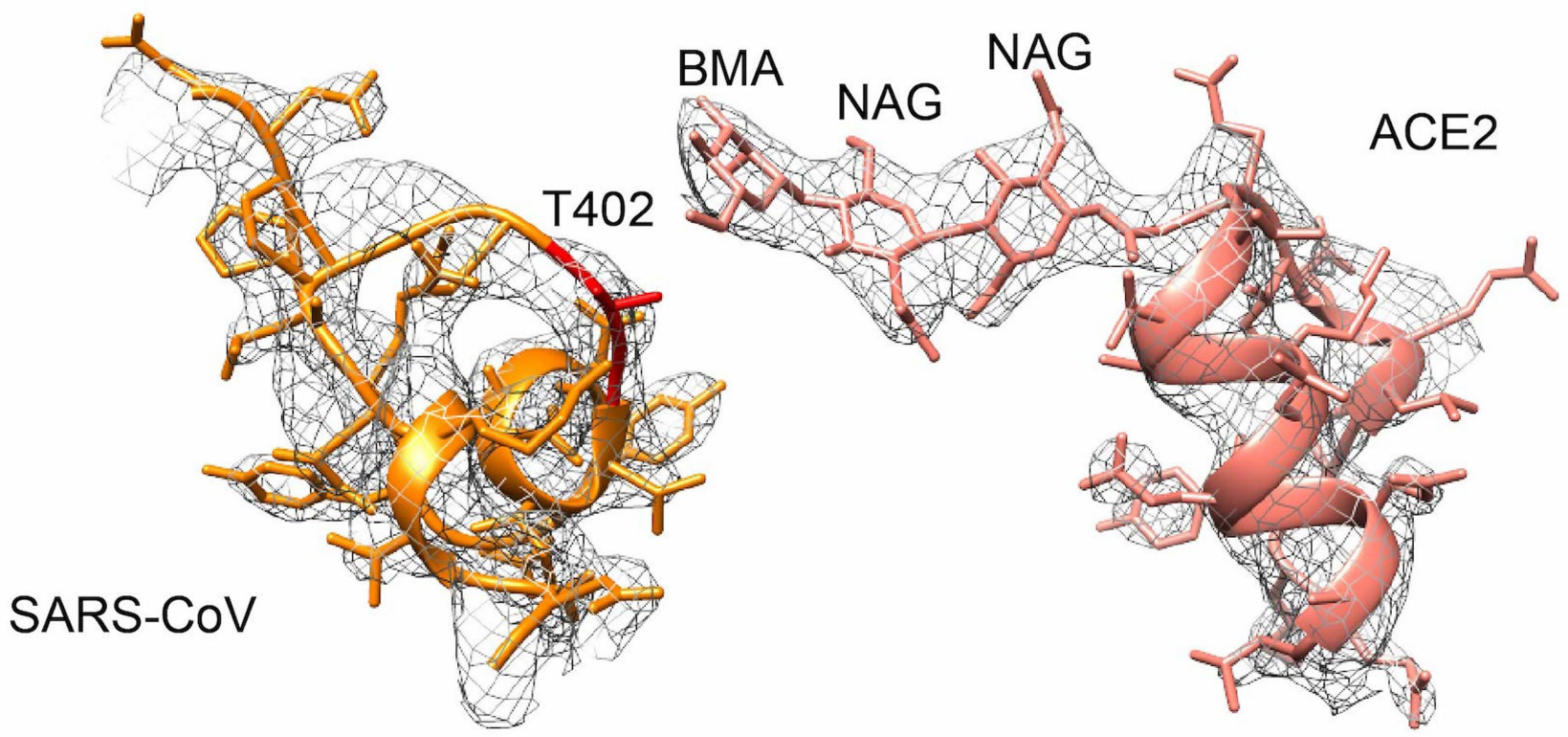

b
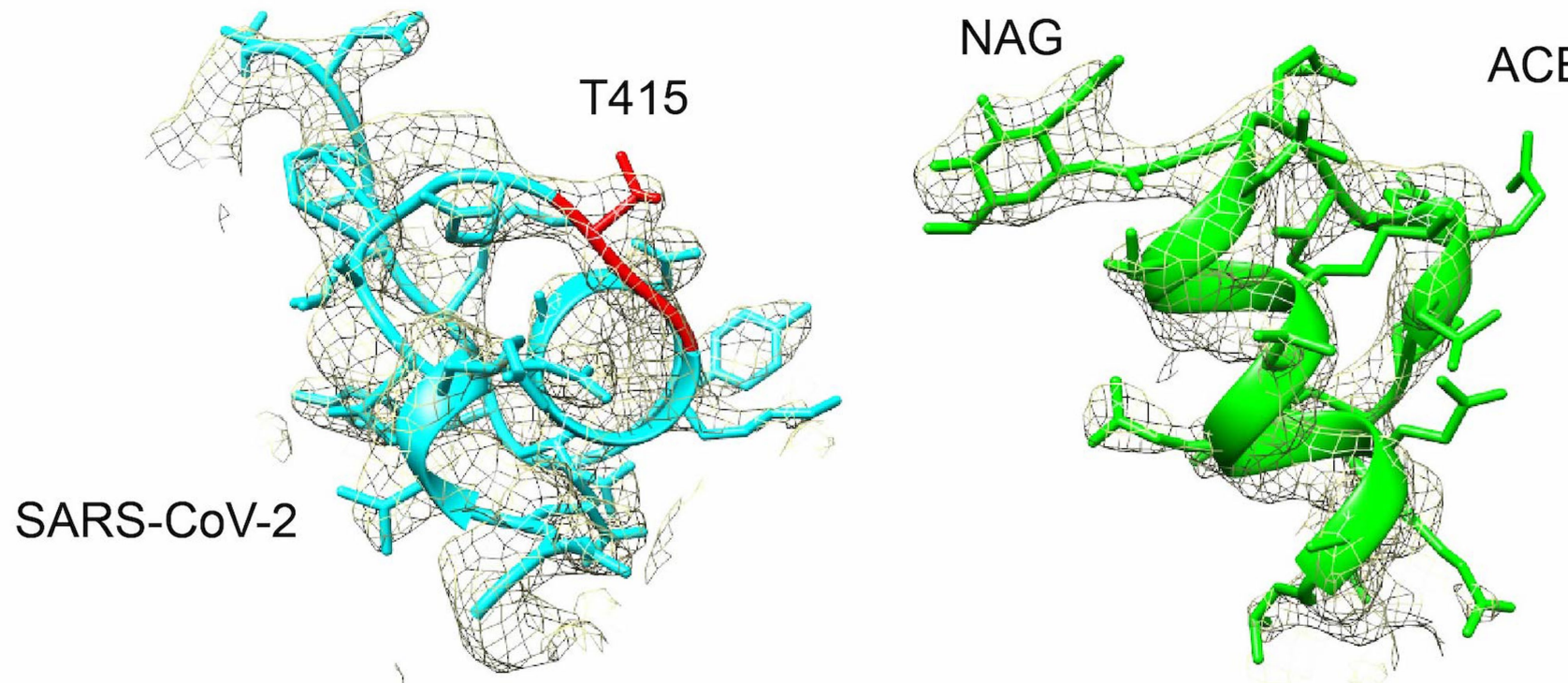

Extended Data Fig. 5 | Asn90-linked glycans of ACE2. a, The interface between Asn90-linked glycans of ACE2 (salmon) and SARS-CoV RBD (orange).b, The interface between Asn90-linked glycan of ACE2 (green) and SARS-CoV-2RBD (cyan). The $2 F_{0}-F_{c}$ electron densities contoured at $1.5 \sigma$ are also shown. 
SARS-CoV RBD-ACE2

\section{Data collection}

Space group

$\mathrm{P} 4{ }_{12}{ }_{12}$

Cell dimensions

$$
\begin{aligned}
& a, b, c(\AA) \\
& \alpha, \beta, \gamma,\left(^{\circ}\right)
\end{aligned}
$$$$
\text { 104.67, 104.67, } 228.72
$$

$90,90,90$

Resolution $(\AA)$

$53.1-2.45(2.54-2.45)$ *

$R_{\text {sym }}$ or $R_{\text {merge }}$

$0.118(2.70)$

I/ $\mathrm{s} \mid$

24.2(1.7)

Completeness (\%)

99.90(99.98)

Redundancy

26.1(27.3)

\section{Refinement}

Resolution ( $\AA$ )

No. reflections

$R_{\text {work }} / R_{\text {free }}$

No. atoms

Protein

Ligand/ion

Water

$B$-factors

Protein

Ligand/ion

Water

R.m.s. deviations

Bond lengths $(\AA)$

Bond angles $\left({ }^{\circ}\right)$
0.008

53.1-2.45

47555

$19.6 / 23.7$

6419

57

80

64.7

91.4

58.1

1.21 


\begin{tabular}{|c|c|c|c|}
\hline $\begin{array}{c}\text { SARS-CoV-2 } \\
\text { RBD }\end{array}$ & ACE2 & $\begin{array}{c}\text { SARS-CoV } \\
\text { RBD }\end{array}$ & ACE2 \\
\hline K417 & Q24 & R426 & Q24 \\
\hline G446 & T27 & Y436 & T27 \\
\hline Y449 & F28 & Y440 & F28 \\
\hline Y453 & D30 & Y442 & K31 \\
\hline L455 & K31 & L443 & H34 \\
\hline F456 & H34 & L472 & E37 \\
\hline A475 & E35 & N473 & D38 \\
\hline F486 & E37 & Y475 & Y41 \\
\hline N487 & D38 & N479 & Q42 \\
\hline Y489 & Y41 & G482 & L45 \\
\hline Q493 & Q42 & Y484 & L79 \\
\hline G496 & L79 & T486 & M82 \\
\hline Q498 & M82 & T487 & Y83 \\
\hline T500 & Y83 & G488 & Q325 \\
\hline N501 & N330 & 1489 & E329 \\
\hline G502 & K353 & Y491 & N330 \\
\hline \multirow[t]{4}{*}{ Y505 } & G354 & & K353 \\
\hline & D355 & & G354 \\
\hline & R357 & & D355 \\
\hline & R393 & & R357 \\
\hline
\end{tabular}




\section{natureresearch}

\section{Reporting Summary}

Nature Research wishes to improve the reproducibility of the work that we publish. This form provides structure for consistency and transparency in reporting. For further information on Nature Research policies, see Authors \& Referees and the Editorial Policy Checklist.

\section{Statistics}

For all statistical analyses, confirm that the following items are present in the figure legend, table legend, main text, or Methods section.

n/a Confirmed

Х $\square$ The exact sample size $(n)$ for each experimental group/condition, given as a discrete number and unit of measurement

Х $\square$ A statement on whether measurements were taken from distinct samples or whether the same sample was measured repeatedly

$\square$ The statistical test(s) used AND whether they are one- or two-sided

$\triangle$ Only common tests should be described solely by name; describe more complex techniques in the Methods section.

$\triangle \square$ A description of all covariates tested

Х $\square$ A description of any assumptions or corrections, such as tests of normality and adjustment for multiple comparisons

$\triangle$ A full description of the statistical parameters including central tendency (e.g. means) or other basic estimates (e.g. regression coefficient)

Х AND variation (e.g. standard deviation) or associated estimates of uncertainty (e.g. confidence intervals)

$\square$ For null hypothesis testing, the test statistic (e.g. $F, t, r$ ) with confidence intervals, effect sizes, degrees of freedom and $P$ value noted

$\triangle \square$ Give $P$ values as exact values whenever suitable.

Х $\square$ For Bayesian analysis, information on the choice of priors and Markov chain Monte Carlo settings

Х $\square$ For hierarchical and complex designs, identification of the appropriate level for tests and full reporting of outcomes

Х $\square$ Estimates of effect sizes (e.g. Cohen's $d$, Pearson's $r$ ), indicating how they were calculated

Our web collection on statistics for biologists contains articles on many of the points above.

\section{Software and code}

Policy information about availability of computer code

Data collection NCBI was used for downloading the published SARS-CoV-2 sequences to do the research

Data analysis Aquarium pipeline, CCP4 v7.0, COOT 0.8.6 and PHENIX 1.15.2-3472 were used at the determination of complex structure for data processing, model building and refinement. PyMOL 1.8.x was used to generate the structural figures.

For manuscripts utilizing custom algorithms or software that are central to the research but not yet described in published literature, software must be made available to editors/reviewers. We strongly encourage code deposition in a community repository (e.g. GitHub). See the Nature Research guidelines for submitting code \& software for further information.

\section{Data}

Policy information about availability of data

All manuscripts must include a data availability statement. This statement should provide the following information, where applicable:

- Accession codes, unique identifiers, or web links for publicly available datasets

- A list of figures that have associated raw data

- A description of any restrictions on data availability

Crystal structure presented in this work has been deposited in the Protein Data Bank (PDB) and are available with accession codes 6MOJ.

\section{Field-specific reporting}

Please select the one below that is the best fit for your research. If you are not sure, read the appropriate sections before making your selection. 


\section{Life sciences study design}

All studies must disclose on these points even when the disclosure is negative.
Sample size
Two separate proteins were purified and formed a complex.
Data exclusions
No data excluded.
Replication
The crystal of the complex were obtained in duplicate. All attempts at replication were successful.
Randomization
N/A
Blinding
N/A

\section{Reporting for specific materials, systems and methods}

We require information from authors about some types of materials, experimental systems and methods used in many studies. Here, indicate whether each material, system or method listed is relevant to your study. If you are not sure if a list item applies to your research, read the appropriate section before selecting a response.

Materials \& experimental systems Methods

$\mathrm{n} / \mathrm{a}$ Involved in the study

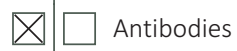

$\square$ Eukaryotic cell lines

\ $\square$ Palaeontology

Х $\square$ Animals and other organisms

\ $\square$ Human research participants

Х $\square$ Clinical data

\section{Eukaryotic cell lines}

Policy information about cell lines

Cell line source(s)

SF9 and Hi5 cells were bought from ATCC

Authentication

SF9 : refered to website: https://www.atcc.org/products/all/CRL-1711.aspx

Hi5: refered to website

These two cell lines are all available in commercial company.

Mycoplasma contamination

We confirm that all cell lines were negative for mycoplasma contamination

Commonly misidentified lines

(See ICLAC register)

No commonly misidentified cell lines were used 